\title{
Model Calibration via Distributionally Robust Optimization: On the NASA Langley Uncertainty Quantification Challenge
}

\author{
Yuanlu Bai \\ Department of Industrial Engineering $\&$ Operations Research, Columbia University, \\ USA. \\ Zhiyuan Huang \\ Department of Management Science $\& 3$ Engineering, Tongji University, Shanghai, China. \\ Henry Lam \\ Department of Industrial Engineering $\&$ Operations Research, Columbia University, \\ USA.
}

\begin{abstract}
We study a methodology to tackle the NASA Langley Uncertainty Quantification Challenge, a model calibration problem under both aleatory and epistemic uncertainties. Our methodology is based on an integration of robust optimization, more specifically a recent line of research known as distributionally robust optimization, and importance sampling in Monte Carlo simulation. The main computation machinery in this integrated methodology amounts to solving sampled linear programs. We present theoretical statistical guarantees of our approach via connections to nonparametric hypothesis testing, and numerical performances including parameter calibration and downstream decision and risk evaluation tasks.
\end{abstract}

Keywords: uncertainty quantification, model calibration, distributionally robust optimization, importance sampling, linear programming, nonparametric.

Email addresses: yb2436@columbia.edu (Yuanlu Bai), zhyhuang@umich.edu (Zhiyuan Huang), henry.lam@columbia.edu (Henry Lam) 
We consider the NASA Langley Uncertainty Quantification (UQ) Challenge problem [16] where, given a set of "output" data and under both aleatory and epistemic uncertainties, we aim to infer a region that contains the true values of the associated variables. These steps allow us to investigate the reduction of uncertainty by obtaining further information and estimate the failure probabilities of related systems. To tackle these challenges, we study a methodology based on an integration of robust optimization (RO), more specifically, a recent line of research known as distributionally robust optimization (DRO), and importance sampling in Monte Carlo simulation. We will see that the main computation machinery in this integrated methodology boils down to solving sampled linear programs (LPs). In this paper, we will explain our methodology, introduce theoretical statistical guarantees via connections to nonparametric hypothesis testing, and present the numerical results on this UQ Challenge.

We briefly introduce the Challenge and notations, where details can be found in [16]. The uncertainty model in the Challenge is given by $\left\langle f_{a}, E\right\rangle$, where $a \sim f_{a}$ is an aleatory variable following a probability density $f_{a}$ and probability distribution function $F_{a}$, and $e \in E$ is an epistemic variable inside the deterministic set $E$. Both the true distribution of $a$ and the true value of $e$ are unknown. Initially, we are given $E_{0} \supset E$ and data $D_{1}=$ $\left\{y^{(i)}(t)\right\}, i=1, \ldots, n_{1}$ in the form of a discrete-time trajectory $t=0, \ldots, T$. We have the computational capability to simulate $y(a, e, t)$ for given values of $a \in A, e \in E_{0}$. The task is to calibrate the distribution of $a$ and value of $e$ with uncertainty quantification, as well as using them to conduct downstream decision and risk evaluation tasks.

\section{Overview of Our Methodology (Problem A)}

We first give a high-level overview of our methodology in extracting a region $E$ that contains the true epistemic variables. For convenience, we call this region an "eligibility set" of $e$. For each value of $e$ inside $E$, we also have a set (in the space of probability distributions) that contains "eligible" distributions for the random variable $a$. For the sake of computational tractability (as we will see shortly), the eligibility set of $e$ is represented by a set of sampled points in $E_{0}$ that approximate its shape, whereas the eligibility set of $a$ is represented by probability weights on sampled points on $A$. The eligibility set $E$ and the corresponding eligibility set of distributions for $a$ are obtained by solving an array of LPs that are constructed 
from these properly sampled points, and then deciding eligibility by checking the LP optimal values against a threshold that resembles the " $p$-value" approach in hypothesis testing. As another key ingredient, this methodology involves a dimension-collapsing transformation $\mathbf{S}$, applied on the raw data, which ultimately allows using the Kolgomorov-Smirnov (KS) statistic to endow rigorous statistical guarantees.

Algorithm 1 is a procedural description of our approach to construct the eligibility set $E$, which also gives as a side product an eligibility set of the distributions of $a$ for each $e$, represented by weights in the set (11). In the following, we explain the elements and terminologies in this algorithm in detail.

Algorithm 1 Constructing eligibility set $E$

Input: Data $D_{1}=\left\{\left(y^{(i)}(t)\right)_{t=0, \ldots, T}\right\}_{i=1, \ldots, n_{1}}$. A uniformly sampled set of $e^{(l)}, l=1, \ldots, n_{2}$ over $E_{0}$. A uniformly sampled set of $a^{(j)}, j=1, \ldots, k$ over A. A summary function $\mathbf{S}(\cdot): \mathbb{R}^{n_{t}+1} \rightarrow \mathbb{R}^{m}$. A target confidence level $1-\alpha$. Procedure:

1. Simulate outputs from the baseline distribution: Evaluate $\left(y\left(a^{(j)}, e^{(l)}, t\right)\right)_{t=0, \ldots, T}$ for $j=1, \ldots, k, l=1, \ldots, n_{2}$.

2. Summarize the outputs: Evaluate $\mathbf{s}^{(i)}=\mathbf{S}\left(\left(y^{(i)}(t)\right)_{t=0, \ldots, T}\right)$ for $i=$ $1, \ldots, n_{1}$, and $\left.\mathbf{S}\left(y\left(a^{(j)}, e^{(l)}, t\right)\right)_{t=0, \ldots, T}\right)$ for $j=1, \ldots, k, l=1, \ldots, n_{2}$.

3. Compute the degree of eligibility: For each $l=1, \ldots, n_{2}$, solve optimization problem Eq. 10 to obtain $q_{l}^{*}$.

4. Construct the eligibility set: Output $E=\left\{e^{(l)}: q_{l}^{*} \leq q_{1-\alpha / m}\right\}$. Smooth the set if needed.

\section{A DRO Perspective}

Our starting idea is to approximate the set

$$
E=\left\{e \in E_{0}: \text { there exists } P_{e} \text { s.t. } d\left(P_{e}, \hat{P}\right) \leq \eta\right\}
$$

where $P_{e}$ is the probability distribution of $\{y(a, e, t)\}_{t=0, \ldots, T}$, namely the outputs of the simulation model $\{y(a, e, t)\}_{t=0, \ldots, T}$ at a fixed $e$ but random $a$. $\hat{P}$ denotes the empirical distribution of $D_{1}$, more concretely the distribution given by

$$
\hat{P}(\cdot)=\frac{1}{n_{1}} \sum_{i=1}^{n_{1}} \delta_{\left(y^{(i)}(t)\right)_{t=0, \ldots, T}}(\cdot)
$$




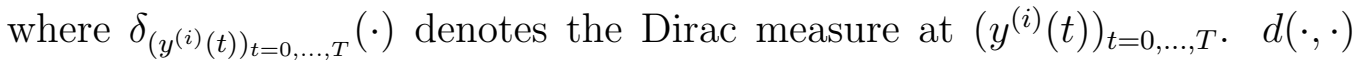
denotes a discrepancy between two probability distributions, and $\eta \in \mathbb{R}_{+}$is a suitable constant. Intuitively, $E$ in Eq. (1) is the set of $e$ such that there exists a distribution for the outputs that is close enough to the empirical distribution from the data. If for a given $e$ there does not exist any possible output distribution that is close to $\hat{P}$, then $e$ is likely not the truth. The following gives a theoretical justification for using Eq. (1):

Theorem 1. Suppose that the true distribution of the output $(y(t))_{t=0, \ldots, T}$, called $P_{\text {true }}$, satisfies $d\left(P_{\text {true }}, \hat{P}\right) \leq \eta$ with confidence level $1-\alpha$, i.e., we have

$$
\mathbb{P}\left(d\left(P_{\text {true }}, \hat{P}\right) \leq \eta\right) \geq 1-\alpha
$$

where $\mathbb{P}$ denotes the probability with respect to the data. Then the set $E$ in Eq. (1) satisfies $\mathbb{P}\left(e_{\text {true }} \in E\right) \geq 1-\alpha$, where $e_{\text {true }}$ denotes the true value of e. Similar deduction holds if Eq. (2) holds asymptotically (as the data size grows), in which case the same asymptotic modification holds for the conclusion.

The proof of Theorem 1 comes from a straightforward set inclusion.

Proof. Note that $d\left(P_{\text {true }}, \hat{P}\right) \leq \eta$ implies $e_{\text {true }} \in E$. Thus we have $\mathbb{P}\left(e_{\text {true }} \in\right.$ $E) \geq \mathbb{P}\left(d\left(P_{\text {true }}, \hat{P}\right) \leq \eta\right) \geq 1-\alpha$. Similar derivation holds for the asymptotic version.

In Eq. (1), the set of distributions $\left\{P_{e}: d\left(P_{e}, \hat{P}\right) \leq \eta\right\}$ is analogous to the so-called uncertainty set or ambiguity set in the RO literature (e.g., [7, 6]), which is a set postulated to contain the true values of uncertain parameters in a model. RO generally advocates decision-making under uncertainty that hedges against the worst-case scenario, where the worst case is over the uncertainty set (and thus often leads to a minimax optimization problem). $\mathrm{DRO}$, in particular, focuses on problems where the uncertainty is on the probability distribution of an underlying random variable (e.g., [62, 18]). This is the perspective that we are taking here, where $a$ has a distribution that is unknown, in addition to the uncertainty on $e$. Moreover, we also take a generalized view of RO or DRO here as attempting to construct an eligibility set of $e$ instead of finding a robust decision via a minimax optimization.

Theorem 1 focuses on the situation where the uncertainty set is constructed and calibrated from data, which is known as data-driven RO or 
DRO ([8, 33]). If such an uncertainty set has the property of being a confidence region for the uncertain parameters or distributions, then by solving $\mathrm{RO}$ or DRO, the confidence guarantee can be translated to the resulting decision, or the eligibility set in our case. Here we have taken a nonparametric and frequentist approach, as opposed to other potential Bayesian methods.

In implementation we choose $\alpha=0.05$, so that the eligibility set $E$ has the interpretation of approximating a $95 \%$ confidence set for $e$. In the above developments, $d\left(P_{e}, \hat{P}\right) \leq \eta$ can in fact be replaced with more general set $P_{e} \in$ $\mathcal{U}$ where $\mathcal{U}$ is calibrated from the data. Nonetheless, the distance-based set (or "ball") surrounding the empirical distribution is intuitive to understand, and our specific choice of the set below falls into such a representation.

To use Eq. (1), there are two immediate questions:

1. What $d(\cdot, \cdot)$ should and can we use, and how do we calibrate $\eta$ ?

2. How do we determine whether there exists $P_{e}$ that satisfies $d\left(P_{e}, \hat{P}\right) \leq \eta$ for a given $e$ ?

In the following two sections, we address the above two questions respectively which would then lead us to Algorithm 1 .

\section{Constructing Discrepancy Measures}

For the first question, we first point out that in theory many choices of $d$ could be used (basically, any $d$ that satisfies the confidence property in Theorem 11. But, a poor choice of $d$ would lead to a more conservative result, i.e., larger $E$, than others. A natural choice of $d$ should capture the discrepancy of the distributions efficiently. Moreover, the choice of $d$ should also account for the difficulty in calibrating $\eta$ such that the assumption in Theorem 1 can be satisfied, as well as the computational tractability in solving the eligibility determination problem in Eq. (1).

Based on the above considerations, we construct $d$ and calibrate $\eta$ as follows. First, we "summarize" the data $D_{1}$ into a lower-dimensional representation, say $\left\{s_{1}^{(i)}, \ldots, s_{m}^{(i)}\right\}, i=1, \ldots, n_{1}$, where $s_{v}^{(i)}=S_{v}\left(y^{(i)}(t)_{t=0, \ldots, T}\right)$ for some function $S_{v}(\cdot)$. For convenience, we denote $\mathbf{S}(\cdot)=\left(S_{1}(\cdot), \ldots, S_{m}(\cdot)\right)$ : $\mathbb{R}^{n_{t}+1} \rightarrow \mathbb{R}^{m}$, and $\mathbf{s}^{(i)}=\left(s_{1}^{(i)}, \ldots, s_{m}^{(i)}\right)$. We call $\mathbf{S}(\cdot)$ the "summary function" and $\mathbf{s}^{(i)}$ the "summaries" of the $i$-th output. $\mathbf{S}(\cdot)$ attempts to capture important characteristics of the raw data (we will see later that we use the positions and values of the peaks extracted from Fourier analysis). Also, the low dimensionality of $\mathbf{s}^{(i)}$ is important to calibrate $\eta$ well. 
Next, we define

$$
d\left(P_{e}, \hat{P}\right)=\max _{v=1, \ldots, m} \sup _{x \in \mathbb{R}}\left|F_{e, v}(x)-\hat{F}_{v}(x)\right|
$$

where $\hat{F}_{v}(x)=\frac{1}{n_{1}} \sum_{i=1}^{n_{1}} I\left(s_{v}^{(i)} \leq x\right)$, with $I(\cdot)$ denoting the indicator function, is the empirical distribution function of $s_{v}^{(i)}$ (i.e., the distribution function of $\hat{P}$ projected onto the $v$-th summary). $F_{e, v}(x)$ is the probability distribution function of the $v$-th summary of the simulation model output $S_{v}(y(a, e, t))_{t=0, \ldots, T}$ (i.e., the distribution function of the projection of $P_{e}$ onto the $v$-th summary). We then choose $\eta=q_{1-\alpha / m} / \sqrt{n_{1}}$ as the $(1-\alpha / m)$ quantile of the Kolmogorov-Smirnov (KS) statistic, namely that $q_{1-\alpha / m}$ is the $(1-\alpha / m)$-quantile of $\sup _{x \in[0,1]}|B B(x)|$ where $B B(\cdot)$ denotes a standard Brownian bridge.

To understand Eq. (3), note that the set of $P_{e}$ that satisfies $d\left(P_{e}, \hat{P}\right) \leq \eta$ is equivalent to $P_{e}$ that satisfies

$$
\sup _{x \in \mathbb{R}}\left|F_{e, v}(x)-\hat{F}_{v}(x)\right| \leq \frac{q_{1-\alpha / m}}{\sqrt{n_{1}}}, \quad v=1, \ldots, m
$$

Here, $\sup _{x \in \mathbb{R}}\left|F_{e, v}(x)-\hat{F}_{v}(x)\right|$ is the KS-statistic for a goodness-of-fit test against the distribution $F_{e, v}(x)$, using the data on the $v$-th summary. Since we have $m$ summaries and hence $m$ tests, we use a Bonferroni correction and deduce that

$$
\liminf _{n_{1} \rightarrow \infty} \mathbb{P}\left(\sup _{x \in \mathbb{R}}\left|F_{\text {true }, v}(x)-\hat{F}_{v}(x)\right| \leq \frac{q_{1-\alpha / m}}{\sqrt{n_{1}}} \text { for } v=1, \ldots, m\right) \geq 1-\alpha
$$

where $F_{\text {true }, v}$ denotes the true distribution function of the $v$-th summary. Thus, the (asymptotic version of the) assumption in Theorem 1 holds.

Note that here the quality of the summaries does not affect the statistical correctness of our method (in terms of overfitting), but it does affect crucially the resulting conservativeness (in the sense of getting a larger $E$ ). Moreover, in choosing the number of summaries $m$, there is a tradeoff between the conservativeness coming from representativeness and simultaneous estimation. On one end, using more summaries means more knowledge we impose on $P_{e}$, which translates into a smaller feasible set for $P_{e}$ and ultimately a smaller eligibility set $E$. This relation, however, is true only if there is no statistical noise coming from the data. In the case of finite data size $n_{1}$, then 
more summaries also means that constructing the feasible set for $P_{e}$ requires more simultaneous estimations in calibrating its size, which is manifested in the Bonferroni correction whose degree increments with each additional summary. In our implementation (see Section 7), we find that using 12 summaries seems to balance well this representativeness versus simultaneous estimation error tradeoff.

\section{Determining Existence of an Aleatory Distribution}

Now we address the second question on how we can decide, for a given $e$, whether a $P_{e}$ exists such that $d\left(P_{e}, \hat{P}\right) \leq \eta$. We first rephrase the representation with a change of measure. Consider a "baseline" probability distribution, say $P_{0}$, that is chosen by us in advance. A reasonable choice, for instance, is the uniform distribution over $A$, the support of $a$. Then we can write $d\left(P_{e}, \hat{P}\right) \leq \eta$ as

$$
\sup _{x \in \mathbb{R}}\left|\int_{S_{v}(u) \leq x} W_{e}(u) d P_{0}(u)-\hat{F}_{v}(x)\right| \leq \frac{q_{1-\alpha / m}}{\sqrt{n_{1}}}
$$

for $v=1, \ldots, m$ where $W_{e}(\cdot)=d P_{e} / d P_{0}$ is the Radon-Nikodym derivative of $P_{e}$ with respect to $P_{0}$, and we have used the change-of-measure representation $F_{e, v}(x)=\int_{S_{v}(u) \leq x} W_{e}(u) d P_{0}(u)$. Here we have assumed that $P_{0}$ is suitably chosen such that absolute continuity of $P_{e}$ with respect to $P_{0}$ holds. Eq. (5) turns the determination of the existence of eligible $P_{e}$ into the existence of an eligible Radon-Nikodym derivative $W_{e}(\cdot)$.

The next step is to utilize Monte Carlo simulation to approximate $P_{0}$. More specifically, given $e$, we run $k$ simulation runs under $P_{0}$ to generate $\left(y\left(a^{(j)}, e, t\right)\right)_{t=0, \ldots, T}$ for $j=1, \ldots, k$. Then Eq. (5) can be approximated by

$$
\sup _{x \in \mathbb{R}}\left|\sum_{j=1}^{k} W_{j} I\left(S_{v}\left(\left(y\left(a^{(j)}, e, t\right)\right)_{t=0, \ldots, T}\right) \leq x\right)-\hat{F}_{v}(x)\right| \leq \frac{q_{1-\alpha / m}}{\sqrt{n_{1}}}, r=1, \ldots, m
$$

where $W_{j}=(1 / k)\left(d P_{e} / d P_{0}\left(\left(y\left(a^{(j)}, e, t\right)\right)\right)\right)$ represents the (unknown) sampled likelihood ratio from the view of importance sampling [36, 12]. Our task is to find a set of weights, $W_{j}, j=1, \ldots, k$, such that Eq. (6) holds. These weights should approximately satisfy the properties of the RadonNikodym derivative, namely positivity and integrating to one. Thus, we seek 
for $W_{j}, j=1, \ldots, k$ such that

$$
\begin{gathered}
\sup _{x \in \mathbb{R}}\left|\sum_{j=1}^{k} W_{j} I\left(S_{v}\left(\left(y\left(a^{(j)}, e, t\right)\right)_{t=0, \ldots, T}\right) \leq x\right)-\hat{F}_{v}(x)\right| \leq \frac{q_{1-\alpha / m}}{\sqrt{n_{1}}}, r=1, \ldots, m \\
\sum_{j=1}^{k} W_{j}=1, W_{j} \geq 0 \text { for } j=1, \ldots, k
\end{gathered}
$$

where Eq. (8) enforces the weights to lie in a probability simplex. If $k$ is much larger than $n_{1}$, then the existence of $W_{j}, j=1, \ldots, k$ satisfying Eq. (7) and Eq. (8) would determine that the considered $e$ is in E. To summarize, we have:

Theorem 2. Suppose $k=\omega\left(n_{1}\right)$, and $P_{\text {true }}$ is absolutely continuous with respect to $P_{0}$ and that $\left\|d P_{\text {true }} / d P_{0}\right\|_{\infty} \leq C$ for some constant $C>0$ and $\|\cdot\|_{\infty}$ denotes the essential supremum. Suppose, for each e, we generate $k$ simulation replications to get $\left.\left(y\left(a^{(j)}, e, t\right)\right)_{t=0, \ldots, T}\right), j=1, \ldots, k$, where $a^{(j)}$ are drawn from $P_{0}$ in an i.i.d. fashion. Then the set

$$
E=\left\{e: \text { there exists } W_{j}, j=1, \ldots, k \text { such that Eq. (7) and Eq. (8) hold }\right\}
$$

will satisfy

$$
\liminf _{n_{1} \rightarrow \infty, k / n_{1} \rightarrow \infty} \mathbb{P}\left(e_{\text {true }} \in E\right) \geq 1-\alpha
$$

The proof is in the appendix. Note that in Theorem 2, $W_{j}$ 's represent the unknown sampled likelihood ratios such that, together with the $a^{(j)}$ 's generated from $P_{0}$, the function

$$
\sum_{j=1}^{k} W_{j} I\left(S_{v}\left(\left(y\left(a^{(j)}, e, t\right)\right)_{t=0, \ldots, T}\right) \leq \cdot\right)
$$

approximates the unknown true $v$-th summary distribution function $F_{t r u e, v}$.

To use the above $E$ and elicit the guarantee in Theorem 2, we still need some steps in order to conduct feasible numerical implementation. First, we need to discretize or sufficiently sample $e$ 's over $E_{0}$, since checking the 
existence of eligible $W_{j}$ 's for all $e$ is computationally infeasible. In our implementation we draw $n_{2}=1000 e$ 's uniformly over $E_{0}$, call them $e^{(1)}, \ldots, e^{\left(n_{2}\right)}$, and then put together the geometry of $E$ from the eligible $e^{(l)}$ 's. Second, the current representation of the KS constraint Eq. (7) involves entire distribution functions. We can write Eq. (7) as a finite number of linear constraints, given by

$$
\begin{aligned}
& \hat{F}_{v}\left(s_{v}^{(i)}+\right)-\frac{q_{1-\alpha / m}}{\sqrt{n_{1}}} \\
\leq & \sum_{j=1}^{k} W_{j} I\left(S_{v}\left(\left(y\left(a^{(j)}, e, t\right)\right)_{t=0, \ldots, T}\right) \leq s_{v}^{(i)}\right) \\
\leq & \hat{F}_{v}\left(s_{v}^{(i)}-\right)+\frac{q_{1-\alpha / m}}{\sqrt{n_{1}}}
\end{aligned}
$$

for $i=1, \ldots, n_{1}, r=1, \ldots, m$ where $s_{v}^{(i)}, i=1, \ldots, n_{1}$ are the $v$-th summary of the $i$-th data point, and $s_{v}^{(i)}+$ and $s_{v}^{(i)}-$ denote the right and left limits of the empirical distribution at $s_{v}^{(i)}$.

Thus, putting everything together, we solve, for each $e^{(l)}, l=1, \ldots, n_{2}$, the feasibility problem:

Find $W_{j}, j=1, \ldots, k$ such that Eq. (9) and Eq. (8) hold.

If there exists feasible $W_{j}, j=1, \ldots, k$, then $e^{(l)}$ is eligible. The set $\left\{e^{(l)}\right.$ : $e^{(l)}$ is eligible $\}$ is an approximation of $E$. Note that this is a "sampled" subset of $E$. In general, without running the simulation at the other points of $E$, there is no guarantee whether these other points are eligible or not. However, if the distribution of $\{y(a, e, t)\}_{t=0, \ldots, T}$ is continuous in $e$ in some suitable sense, then it is reasonable to believe that the neighborhood of an eligible point $e^{(l)}$ is also eligible (and vice versa). In this case, we can "smooth" the discrete set of $\left\{e^{(l)}: e^{(l)}\right.$ is eligible $\}$ if needed (e.g., by doing some clustering and taking the convex hull of each cluster). Finally, note that the feasibility problem above is a linear problem in the decision variables $W_{j}$ 's.

\section{Towards the Main Procedure}

To link to our main Algorithm 1, we offer an equivalent approach to the above feasibility-problem-based procedure that allows further flexibility in choosing the threshold $q_{1-\alpha / m}$, which currently is set as the Bonferroniadjusted KS critical value. This equivalent approach leaves this choice of 
threshold open and can determine the set of eligible $e^{(l)}$ as a function of the threshold, thus giving some room to improve conservativeness should the formed approximate $E$ turns out to be too loose according to other expert opinion. Here, we solve, for each $e^{(l)}, l=1, \ldots, n_{2}$, the optimization problem

$$
\begin{aligned}
q_{l}^{*}=\min & q \\
\text { s.t. } & \hat{F}_{v}\left(s_{v}^{(i)}+\right)-\frac{q}{\sqrt{n_{1}}} \\
& \leq \sum_{j=1}^{k} W_{j} I\left(S_{v}\left(\left(y\left(a^{(j)}, e^{(l)}, t\right)\right)_{t=0, \ldots, T}\right) \leq s_{v}^{(i)}\right) \\
& \leq \hat{F}_{v}\left(s_{v}^{(i)}-\right)+\frac{q}{\sqrt{n_{1}}} \text { for } i=1, \ldots, n_{1}, v=1, \ldots, m ; \\
& \sum_{j=1}^{k} W_{j}=1, W_{j} \geq 0 \text { for } j=1, \ldots, k
\end{aligned}
$$

where the decision variables are $W_{j}, j=1, \ldots, k$ and $q$. If the optimal value $q_{l}^{*}$ satisfies $q_{l}^{*} \leq q_{1-\alpha / m}$, then $e^{(l)}$ is eligible (This can be seen by checking its equivalence to the feasibility problem via the monotonicity of the feasible region for $W_{j}$ 's in Eq. (10) as $q$ increases). The rest then follows as above that $\left\{e^{(l)}: e^{(l)}\right.$ is eligible $\}$ is an approximation of $E$. Like before, Eq. (10) is an LP. Moreover, here $q_{l}^{*}$ captures in a sense the "degree of eligibility" of $e^{(l)}$, and allows convenient visualization by plotting $q_{l}^{*}$ against $e^{(l)}$ to assess the geometry of $E$. For these reasons we prefer to use Eq. (10) over the feasibility problem before. These give the full procedure in Algorithm 1. Note that Algorithm 1 has a variant where we re-generate a sample of $a^{(j)}$ 's for each different $e^{(l)}$. It is clear that the correctness guarantee (Theorem 2 ) still holds in this case.

Moreover, we also present how to find eligible distributions of $a$ for an eligible $e^{(l)}$. The set of eligible distributions of $a$ is approximated by the weights $W_{j}$ 's that satisfy Eq. (9) and Eq. (8), namely

$$
\begin{aligned}
& \left\{W_{j}, j=1, \ldots, k: \hat{F}_{v}\left(s_{v}^{(i)}+\right)-\frac{q_{1-\alpha / m}}{\sqrt{n_{1}}}\right. \\
& \leq \sum_{j=1}^{k} W_{j} I\left(S_{v}\left(\left(y\left(a^{(j)}, e^{(l)}, t\right)\right)_{t=0, \ldots, T}\right) \leq s_{v}^{(i)}\right) \\
& \leq \hat{F}_{v}\left(s_{v}^{(i)}-\right)+\frac{q_{1-\alpha / m}}{\sqrt{n_{1}}}, \text { for } i=1, \ldots, n_{1}, v=1, \ldots, m ; \\
& \left.\sum_{j=1}^{k} W_{j}=1, W_{j} \geq 0 \text { for } j=1, \ldots, k\right\}
\end{aligned}
$$


where $W_{j}$ is the probability weight on $a^{(j)}$. From this, one could also obtain approximate bounds for quantities related to the distribution of $a$. For instance, to get approximate bounds for the mean of $a$, we can maximize and minimize $\sum_{j} W_{j} a^{(j)}$ subject to constraint 11 .

\section{Related Literature}

Before we discuss our numerical findings, we discuss some related literature on the problem setting and our proposed methodology.

The model calibration problem that infers input from output data has been studied across different disciplines. In scientific areas it is viewed as an inverse problem [60], in which Bayesian methodologies are predominantly used (e.g., [17, 15, 37, 3, 32]). Our presented approach is an alternative to Bayesian methods that aim to provide frequentist guarantees in the form of confidence regions. This approach is motivated from the need of sophisticated techniques such as approximate Bayesian computation [48] in the Bayesian framework, and that the DRO methodology that we develop appears to be well-suited to the UQ Challenge setup. In addition to Bayesian approaches, other alternative methods include entropy maximization [39] that use the entropy as a criterion to select the "best" distribution, but it does not have the frequentist guarantee in recovering the true distribution that we provide in this UQ Challenge.

We point out that model calibration has also been investigated in the stochastic simulation community [55, 38]. In this setting, model calibration is often viewed together with model validation. To validate a model, the conventional approach is to use statistical tests such as the two-sample meandifference tests [2] or others like the Schruben-Turing test [57] that decides whether the simulated output data and historical real output data are close enough. If not, then the simulation model is re-calibrated, and this process is repeated until the gap between simulation and real data is sufficiently close. Though having a long history, the development of rigorous frameworks to conduct model calibration and validation has been quite open with relatively few elaborate discussions in the literature [49].

In terms of methodology, our approach is closely related to RO, which is an established method for optimization under uncertainty that advocates the representation of unknown or uncertain parameters in the model as a (deterministic) set (e.g., [7, 6]). This set is often called an uncertainty set 
or an ambiguity set. In the face of decision-making, RO optimizes the decision over the worst-case scenario within the uncertainty set, which usually comes in the form of a minimax problem with the outer optimization on the decision while the inner optimization on the worst case scenario. DRO, a recently active branch of $\mathrm{RO}$, considers stochastic optimization where the underlying probability distribution is uncertain (e.g., [30, 62, 18]). In this case, the uncertainty set lies in the space of probability distributions and one attempts to make decisions under the worst-case distribution. In this paper we take a generalized view of RO or DRO as attempting to find a set of eligible "decisions", namely the $e$, so it does not necessarily involve a minimax problem but instead a set construction.

In data-driven $\mathrm{RO}$ or $\mathrm{DRO}$, the uncertainty set is constructed or calibrated from data. If such a set has the property of being a confidence region for the uncertain parameters or distributions, then by solving the $\mathrm{RO}$ or DRO, the confidence guarantee can be translated to bounds on the resulting decision, and in our case the eligibility set. This approach of constructing uncertainty sets, by viewing them as confidence regions or via hypothesis testing, has been the main approach in data-driven RO or DRO [9]. Recently, alternate approaches have been studied to reduce the conservativeness in set calibration, by utilizing techniques from empirical likelihood [45, 43, 19], Wasserstein profile function [11], Bayesian perspectives [31] and data splitting [33, 44].

In our development, we have constructed an uncertainty set for the unknown distribution $P_{e}$ via a confidence region associated with the KS goodnessof-fit test. This uncertainty set has been proposed in [10]. Other distancebased uncertainty sets, including $\phi$-divergence [51, 5, 27, 41, 42, 4] and Wasserstein distance [20, 13, 22], have also been used, as well as sets based on moment [18, 24, 34] or distributional shape information [52, 46, 61]. We use a simultaneous group of KS statistics with Bonferroni correction, motivated by the tractability in the resulting integration with the importance weighting. The closest work to our framework is the stochastic simulation inverse calibration problem studied in [29], but they consider single-dimensional output and parameter to calibrate the input distributions, in contrast to our "summary" approach via Fourier analysis and the multi-dimensional settings we face.

Another important ingredient in our approach is importance sampling. This is often used as a variance reduction tool (e.g., [58, 28]; [1] Chapter 5; [26] Chapter 4) and is shown to be particularly effective in rare-event sim- 
ulation (e.g., [14, 54, 36, 12]). It operates by sampling a random variable from a different distribution from the true underlying distribution, and applies a so-called likelihood ratio to de-bias the resulting estimate. Other than variance reduction, importance sampling is also used in risk quantification in operations research and mathematical finance that uses a robust optimization perspective (e.g., [27, 25, 41]), which is more closely related to our use in this paper. Additionally, it is used in Bayesian computation [47], and more recently in machine learning contexts such as covariate shift estimation [50, 59] and off-policy evaluation in reinforcement learning [53, 56].

In the remainder of this paper, we illustrate the use of our methodology and report our numerical results on the UQ Challenge.

\section{Summarizing Discrete-Time Histories using Fourier Transform}

By observing the plot of the outputs $y^{(i)}, i=1, \ldots, n_{1}$ (see Fig. 1), we judge that these time series are highly seasonal. Naturally, we choose to use Fourier transform to summarize $(y(t))_{t=0, \ldots, T}$, and we may write $y(t)$ in the form $y(t)=\sum_{k=-\infty}^{\infty} C_{k} e^{-i k \omega_{0} t}$.

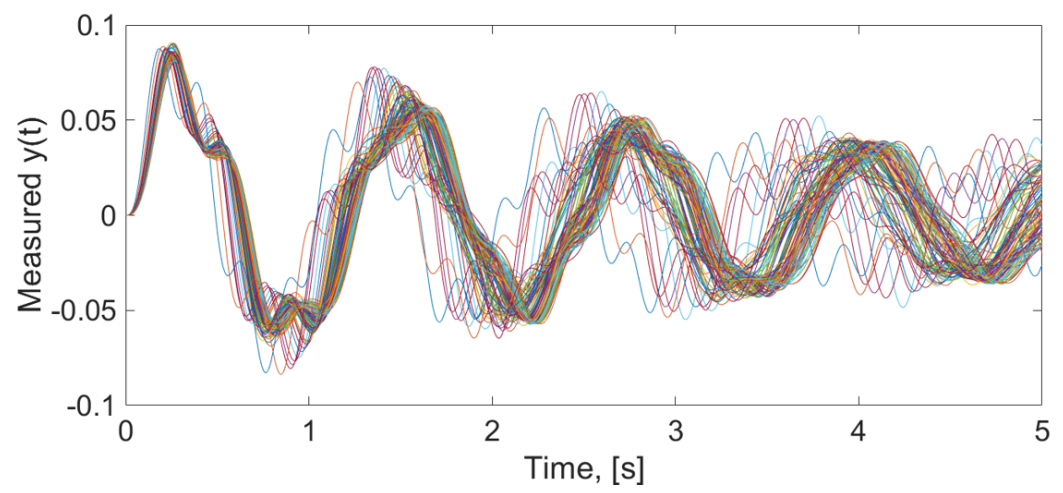

Figure 1: The plot of $y^{(i)}, i=1, \ldots, n_{1}$

First we apply Fourier transform to $y^{(i)}, i=1, \ldots, n_{1}$. For each $y^{(i)}$, we compute the $C_{k}$ 's. Fig. 2 shows the real part and the imaginary part of $C_{k}$ 's against the corresponding frequencies.

For the real part, we see that there is a large positive peak, a large negative peak, a small positive peak and a small negative peak. After testing, we confirm that for any $i$, the large peaks lie in the first 14 terms (from $0 \mathrm{~Hz}$ to 


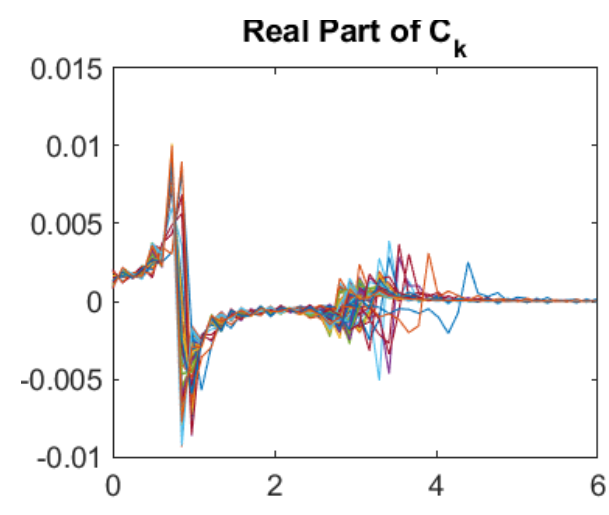

(a) Real part

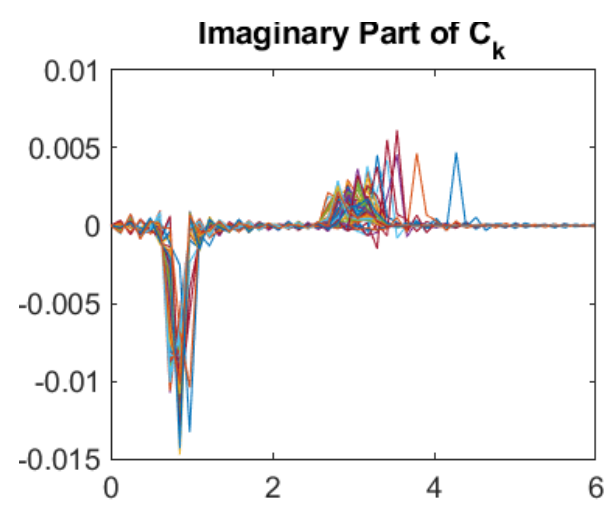

(b) Imaginary part

Figure 2: The real part and the imaginary part of $C_{k}$ 's against the corresponding frequencies

$1.59 \mathrm{~Hz})$, while the small peaks lie between the 15 th term and the 50 th term (from $1.71 \mathrm{~Hz}$ to $5.98 \mathrm{~Hz}$ ). For the imaginary part, we see that there is a large negative peak and a small positive peak. The large peak is also located in the first 14 terms and the small peak between the 15th term and the 50th one.

Therefore, we choose to use the following method to summarize $y$ (i.e., construct the function $\mathbf{S}(\cdot)$ ): first, we apply the Fourier transform to compute $C_{k}$ 's and the corresponding frequencies; second, we compute the real part and the imaginary part of $C_{k}$ 's; third, for the real part, we find the maximum value and the minimum value over $[0 \mathrm{~Hz}, 1.59 \mathrm{~Hz}]$ and $[1.71 \mathrm{~Hz}, 5.98 \mathrm{~Hz}]$, as well as their corresponding frequencies; fourth, for the imaginary part, we find the minimum value over $[0 \mathrm{~Hz}, 1.59 \mathrm{~Hz}]$ and the maximum value over $[1.71 \mathrm{~Hz}, 5.98 \mathrm{~Hz}]$ as well as their corresponding frequencies. Then we use these 12 parameters as the summaries of $y$.

To illustrate how well these summaries fit $y$, Fig. 3(a) shows the comparison for $y^{(1)}$. The fit qualities of other time series are similar to this example. Though they are not extremely close to each other, the fitted curves do resemble the original curves. Note that it is entirely possible to improve the fitting if we keep more frequencies even if they are not as significant as the main peaks. For instance, Fig. 3(b) shows the improved fitting curve if for both real part and imaginary part, we respectively keep the 20 frequencies with the largest values. It can be seen that now the fit quality is quite good. On the other hand, as discussed in Section 2, using a larger number of summaries both represents more knowledge of $P_{e}$ (better fitting) but also leads 
to more simultaneous estimation error when using the Bonferroni correction needed in calibrating the set for $P_{e}$. To balance the conservativeness of our approach coming from representativeness versus simultaneous estimation, we choose to use the 12-parameter summaries depicted before.

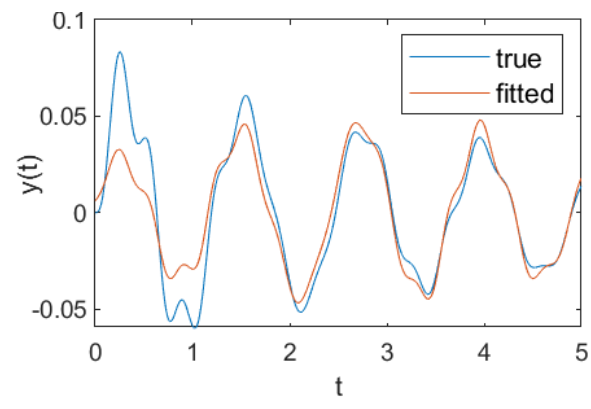

(a) 12 parameters

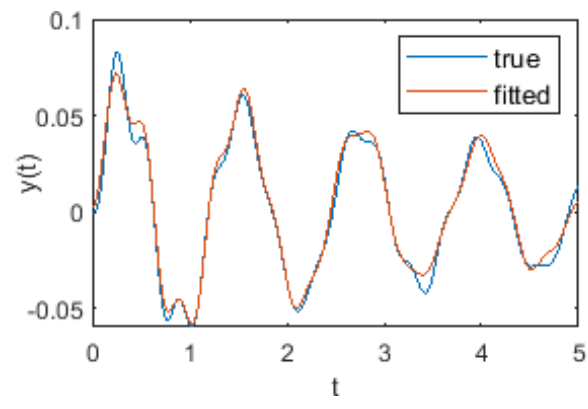

(b) 80 parameters

Figure 3: Fitting $y^{(1)}$ with different number of parameters

\section{Uncertainty Reduction (Problem B)}

\subsection{Ranking Epistemic Parameters (B.1 and B.2)}

Now we implement Algorithm 1 with $n_{2}=k=1000$ and the summary function $\mathbf{S}(\cdot)$ defined in the previous section. The dimension of the summary function is $m=12$. We choose $\alpha$ to be 0.05 . Thus, following the algorithm, for each $l=1, \ldots, n_{2}$, we compute $q_{l}^{*}$ and then compare it with $q_{1-\alpha / m}=$ $q_{1-0.05 / 12}=1.76$.

In Fig. 4, we plot the $q_{l}^{*}$ 's against each dimension of $e$. The red horizontal lines in the graphs correspond to $q_{1-\alpha / m}=1.76$. Thus the dots below the red lines constitute the eligible $e$ 's. We rank the epistemic parameters according to these graphs, namely we rank higher the parameter whose range can potentially be reduced the most. Note that this ranking scheme can be summarized using more rigorous metrics related to the expected amount of eligible $e$ 's after range shrinkage, but since there are only four dimensions, using the graphs directly seem sufficient for our purpose here.

We find that the values of $e_{2}$ and $e_{4}$ of the eligible $e$ 's broadly range from 0 to 2, which implies that reducing the ranges of these two dimensions could hardly reduce our uncertainty. By contrast, the values of $e_{1}$ and $e_{3}$ of the eligible $e$ 's are both concentrated in the lower part of $[0,2]$. Thus, our 
ranking of the epistemic parameters according to their ability to improve the predictive ability is $e_{3}>e_{1}>e_{2}>e_{4}$.

Chances are that the true values of $e_{1}$ and $e_{3}$ are relatively small. In order to further pinpoint the true values of $e_{1}$ and $e_{3}$, we choose to make two uncertainty reductions: increase the lower limits of the bounding interval of $e_{1}$ and $e_{3}$.

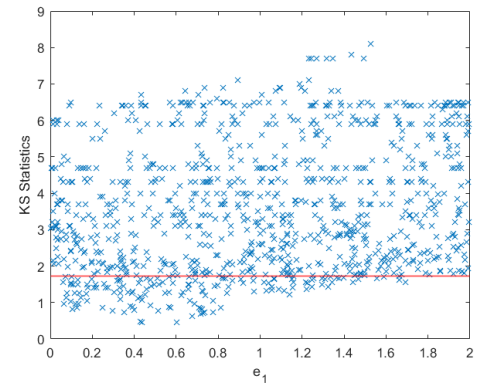

(a) $e_{1}$

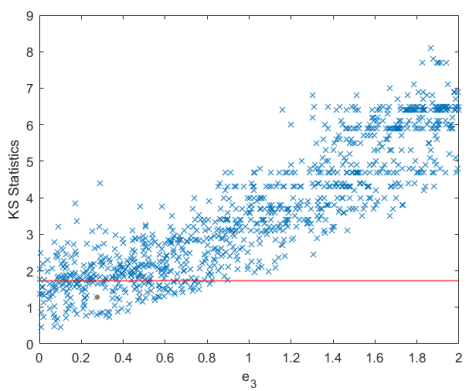

(c) $e_{3}$

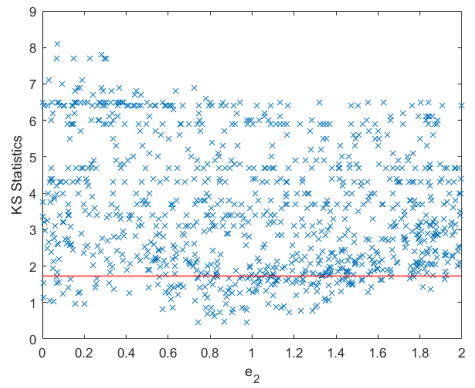

(b) $e_{2}$

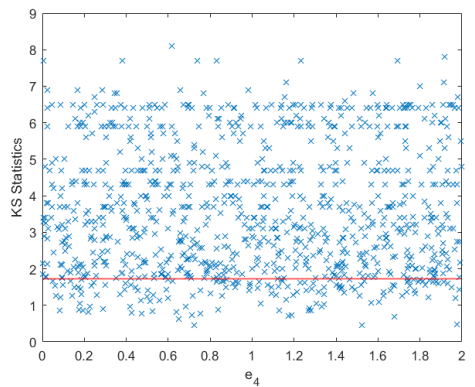

(d) $e_{4}$

Figure 4: $q_{l}^{*}$ against each epistemic variable

\subsection{Impact of the value of $n_{1}$ (A.2)}

To investigate the impact of the value of $n_{1}$, for different values of $n_{1}$ we randomly sample $n_{1}$ outputs without replacement. Then we take these outputs as the new data set. By repeatedly implementing Alg. 1, we find that the larger is $n_{1}$, the smaller is the proportion of eligible $e$ 's. It is intuitive that as the data size grows, $e$ can be better pinpointed. Moreover, except for $e_{4}$, the range of each epistemic variable of eligible $e$ 's obviously shrinks as 
$n_{1}$ increases, which further confirms that $e_{4}$ is the least important epistemic variable.

\subsection{Updated Parameter Ranking (B.3)}

After the epistemic space is reduced, we repeat the process in Section 8.1 but now $e$ 's are generated uniformly from $E_{1}$. From the associated scatter plots (Fig. 5), the updated ranking of the epistemic parameters is $e_{2}>e_{3}>$ $e_{1}>e_{4}$.

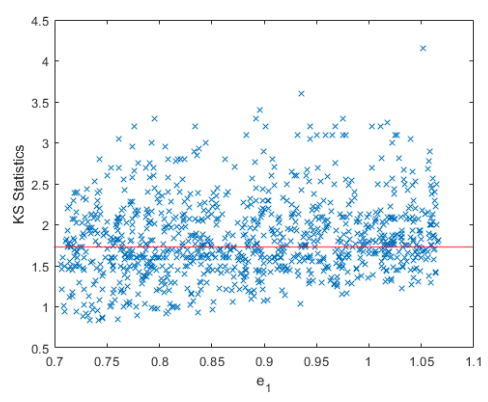

(a) $e_{1}$

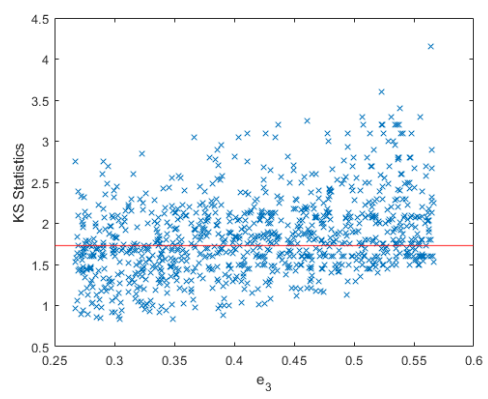

(c) $e_{3}$

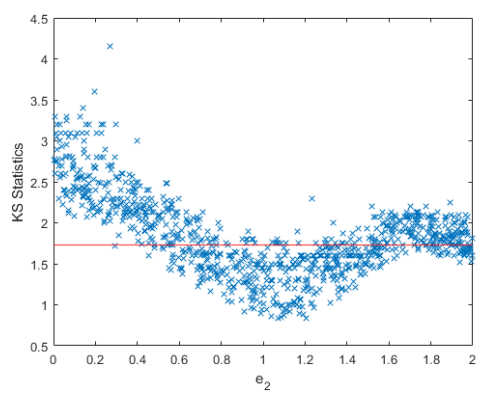

(b) $e_{2}$

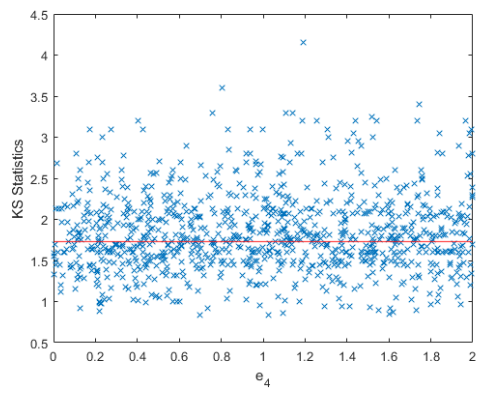

(d) $e_{4}$

Figure 5: $q_{l}^{*}$ against each epistemic variable (refined)

\section{Reliability of Baseline Design (Problem C)}

\subsection{Failure Probabilities and Severity (C.1, C.2 and C.5)}

Combining the refined range of $e$ provided by the host with our Algorithm 1. we construct $E \subset E_{1}$. To estimate $\min _{e \in E} / \max _{e \in E} \mathbb{P}\left(g_{i}(a, e, \theta) \geq 0\right)$, we 
run simulations to respectively solve

$$
\begin{gathered}
\min / \max \sum_{j=1}^{k} W_{j} I\left(g_{i}\left(a^{(j)}, e, \theta\right) \geq 0\right) \\
\text { s.t. } e \in E, W \in U
\end{gathered}
$$

where $U$ is the set of $\left(W_{1}, \cdots, W_{k}\right)$ in Eq. (11). These give the range of $R_{i}(\theta)$. We use the same method to approximate $R(\theta)$, the failure probability for any requirement. Note that in our implementation the $E$ in the formulations above is represented by discrete points $e^{(l)}$ 's. As discussed previously, under additional smoothness assumptions, we could "smooth" these points to obtain a continuum. Nonetheless, under sufficient sampling of $e^{(l)}$, the discretized set should be a good enough approximation in the sense that the optimal values from the "discretized" problems are close to those using the continuum.

Using the above method, we get that the ranges of $R_{1}(\theta), R_{2}(\theta), R_{3}(\theta)$ and $R(\theta)$ are approximately [0, 0.6235], [0, 0.7320], [0, 0.5270] and [0, 0.8217]. Though the ranges seem to be quite wide, they can provide us useful information to be utilized next.

To evaluate $s_{i}(\theta)$, the severity of each individual requirement violation, similarly we simulate

$$
\max _{e \in E} \max _{W \in U} \sum_{j=1}^{k} W_{j} g_{i}\left(a^{(j)}, e, \theta\right) I\left(g_{i}\left(a^{(j)}, e, \theta\right) \geq 0\right) .
$$

The results for $s_{1}(\theta), s_{2}(\theta)$ and $s_{3}(\theta)$ are respectively $0.1464,0.0493$ and 3.5989. Clearly the violation of $g_{3}$ is the most severe one while the violation of $g_{2}$ is the least.

\subsection{Rank for Uncertainties (C.3)}

Our analysis on the rank for epistemic uncertainties is based on the range of $R(\theta)$ obtained above. In our computation, we obtain

$$
\min _{W \in U} / \max _{W \in U} \sum_{j=1}^{k} W_{j} I\left(g_{i}\left(a^{(j)}, e, \theta\right) \geq 0 \text { for some } i=1,2,3\right)
$$

for each eligible $e \in E$. For simplicity, we use $R_{\min }$ and $R_{\max }$ to denote these two values for each eligible $e \in E$ respectively. 
Our approach is to scrutinize the plots of $R_{\min }$ and $R_{\max }$ against each epistemic variable (Fig. 6 and 7). For $R_{\min }$, large value is notable, since it means that any distribution that provides similarity to the original data is going to fail with large probability. Therefore the most ideal reduction is to avoid the region of $e$ such that all $R_{\text {min }}$ 's are large. For $R_{\text {max }}$, the largest $R_{\max }$ for the region denotes the maximum failure probability that one can have. So we pay attention to the epistemic variables that could potentially reduce the "worst-case" failure probability. Based on these considerations, we conclude that the rank for epistemic uncertainties is $e_{3}>e_{1}>e_{2}>e_{4}$.
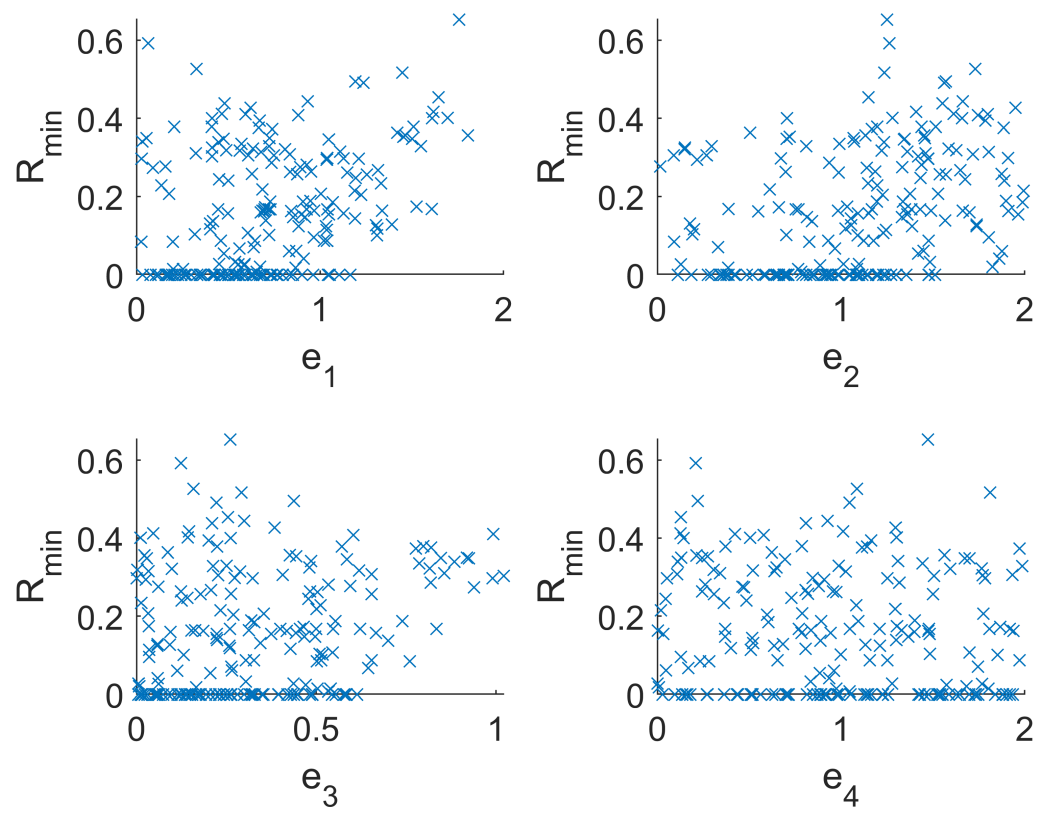

Figure 6: $R_{\min }$ against each epistemic variable.

\subsection{Representative Realizations (C.4)}

Since the distribution of $a$ in our approach is defined as an ambiguity set that depends on $e$, the failure domain would also be based on each eligible $e$. We classify an eligible $e$ to be notable if its corresponding $R_{\min }$ is relatively large (e.g., > 0.1). For convenience, we denote the "best-case" distribution 

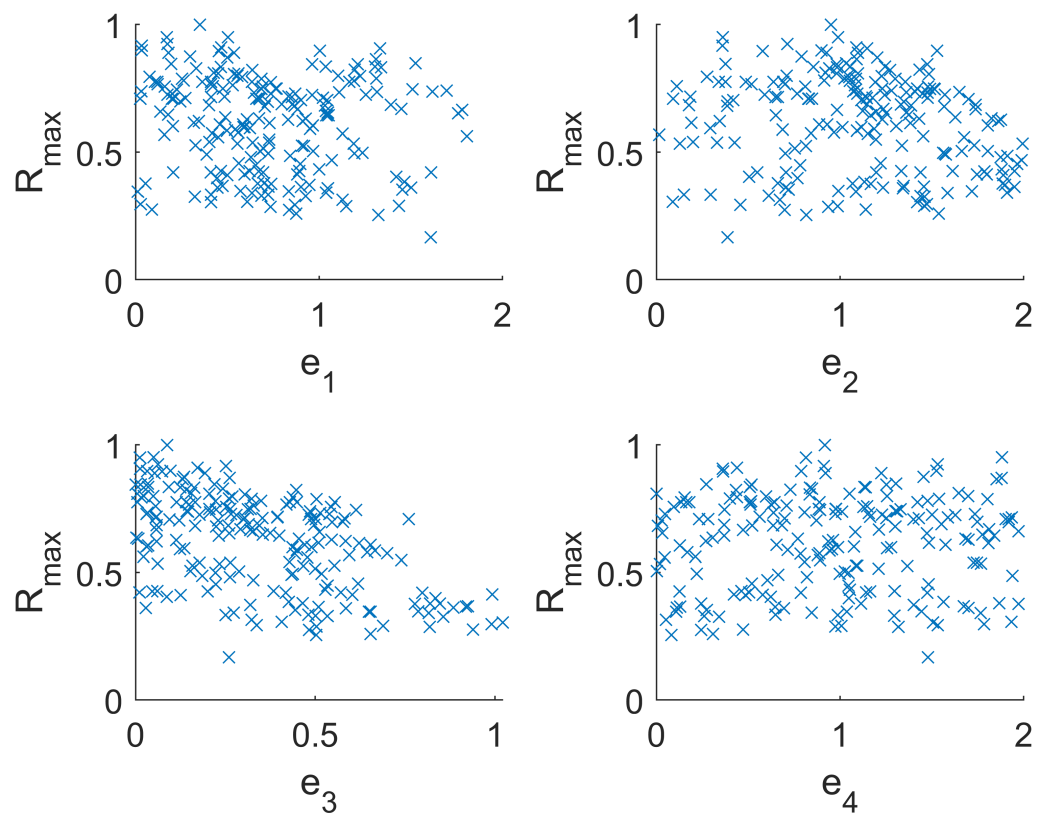

Figure 7: $R_{\max }$ against each epistemic variable.

corresponding to $R_{\min }$ as $w_{\min }$, where

$$
w_{\text {min }}=\arg \min _{W \in U} \sum_{j=1}^{k} W_{j} I\left(g_{i}\left(a^{(j)}, e, \theta\right) \geq 0\right) .
$$

We consider the representative realizations of uncertainties as those $a$ 's with large value of $w_{\min }$ (in our case we consider $>0.05$ ).

From our observation, we find that these representative realizations have a clear pattern on the scatter plot with $a_{1}$ and $a_{3}$ as the coordinates (as in Fig. 8). We also provide some example responses of cases in each group. We observe that there is a clear similarity in the responses within each group, which can be interpreted as different failure patterns.

\section{Reliability-Based Design (Problem D)}

To find a reliability-optimal design point $\theta_{\text {new }}$, we minimize

$$
\max _{e \in E} \min _{W \in U} \sum_{j=1}^{k} W_{j} I\left(g\left(a^{(j)}, e, \theta\right) \geq 0\right) .
$$




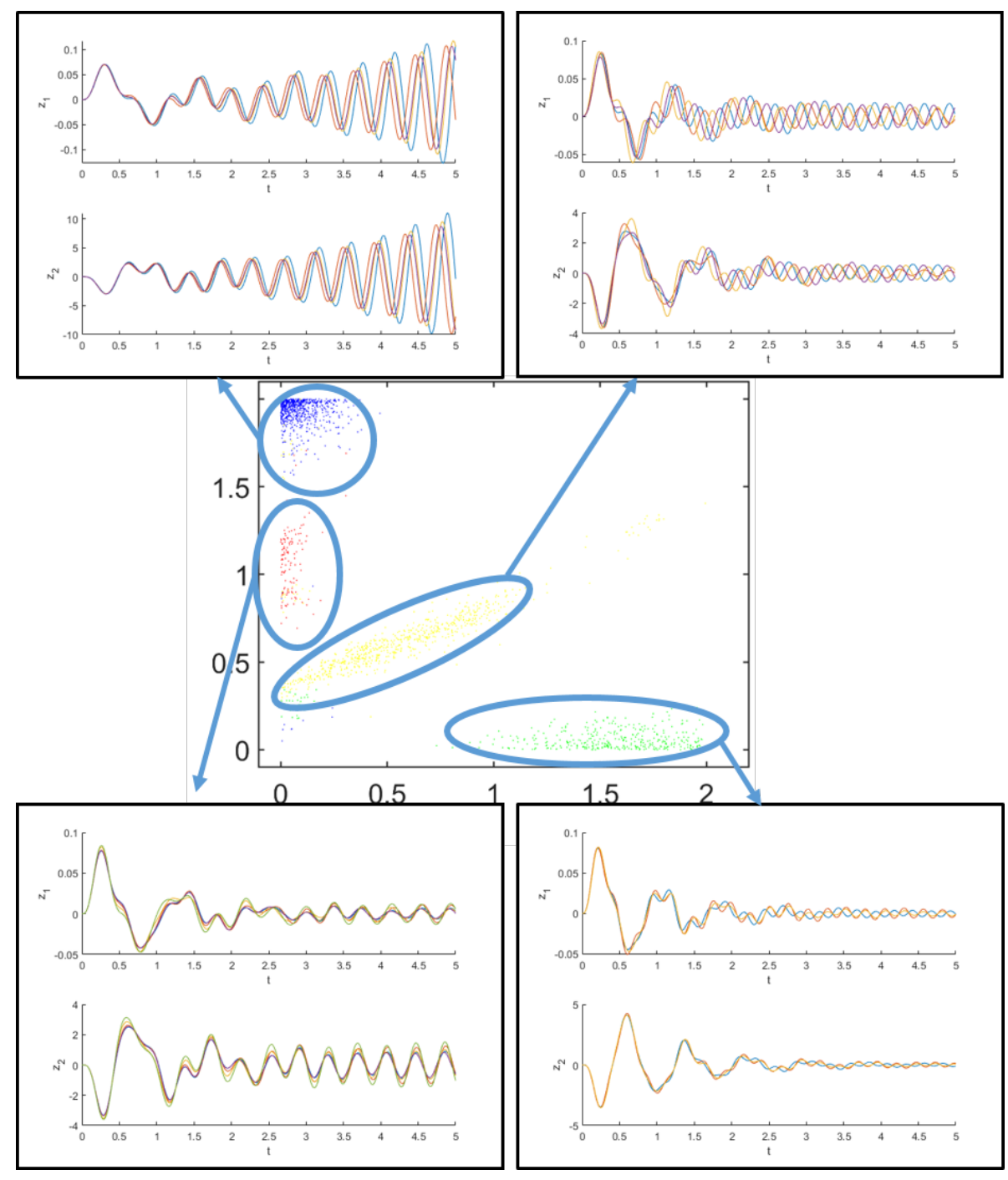

Figure 8: The four groups of representative realizations on the scatter plot with $a_{1}$ and $a_{3}$ as the coordinates. The four groups are failure cases caused by $g_{1}, g_{2}$ and $g_{3}$ (blue), $g_{1}$ (red), $g_{2}$ (yellow) and $g_{3}$ (green). 
Here is the reason why we choose this function as the objective. For an eligible $e \in E$, if $\min _{W \in U} \sum_{j=1}^{k} W_{j} I\left(g\left(a^{(j)}, e, \theta\right) \geq 0\right)$ is large, then the true probability in which the system fails must be even larger than this "best-case" estimate, which implies that this point $e$ has a considerable failure likelihood. The objective above thus aims to find a design point to minimize this best-case estimate, but taking the worst-case among all the eligible $e$ 's. Arguably, one can use other criteria such as minimizing $\max _{e \in E} \max _{W \in U} \sum_{j=1}^{k} W_{j} I\left(g\left(a^{(j)}, e, \theta\right) \geq 0\right)$, but this could make our procedure more conservative.

The optimization problem (13) is of a "black-box" nature since the function $g$ is only observed through simulation, and the problem is easily nonconvex. Our approach is to use a gradient descent to guide us towards a better $\theta_{\text {new }}$, with a goal of finding a reasonably good $\theta_{\text {new }}$ (instead of insisting on full optimality which could be difficult to achieve in this problem). Note that we need to sample $a^{(j)}$ when we land at a new $\theta$ during our iterations, and hence our approach takes the form of a stochastic gradient descent or stochastic approximation [21, 35]. Moreover, the gradient cannot be estimated in an unbiased fashion as we only have black-box function evaluation, and thus we need to resort to the use of finite-difference. This results in a zeroth-order or the so-called Kiefer-Wolfowitz (KW) algorithm [40, 23. As we have a nine-dimensional design variable, we choose to update $\theta$ via a coordinate descent, namely at each iteration we choose one of the dimensions and run a central finite-difference along that dimension, followed by a movement of $\theta$ guided by this gradient estimate with a suitable step size. The updates are done in a round-about fashion over the dimensions. The perturbation size in the finite-difference is chosen of order $1 / n^{1 / 4}$ here as it appears to perform well empirically (though theoretically other scaling could be better).

Algorithm 2 shows the details of our optimization procedure. Considering that the components of $\theta_{\text {baseline }}$ are of very different magnitudes, we first perform a normalization to ease this difference. The quantity $x_{n o w}$ encodes the position of the normalized $\theta_{\text {now }}$, and $1_{9}$ denotes a nine-dimensional vector of 1 's that is set as the initial normalized design point. We set $c_{0}=a_{0}=0.1$ and $N_{\max }=8$.

After running the algorithm, we arrive at a new design point, $\theta_{\text {new }}$ : (-0.1999, -0.6975, 315.31, 4525.3, 4924.2, 1.0358, 280.32, 14.171, 132.52). Compared with the baseline design, the objective function decreases from 0.3656 to 0.2732 . Note that this means that the best-case estimate of the 


\begin{tabular}{l}
\hline Algorithm $2 \mathrm{KW}$ algorithm to find $\theta_{\text {new }}$ \\
Input: The baseline design point $\theta_{\text {baseline }}$. The initial step size $c_{0}$. The \\
initial perturbation size $a_{0}$. The max iteration $N_{\text {max }}$. The objective function \\
$f(\theta)$.
\end{tabular}

\section{Procedure:}

Set $x_{n o w}=1_{9}$ and $n=1$.

while $n \leq N_{\max }$ do

Set $c_{n}=c_{0} / n^{1 / 4}$ and $a_{n}=a_{0} / n$.

for $i$ from 1 to 9 do

$$
\begin{aligned}
& u=f\left(\theta_{\text {baseline }} \circ\left(x_{\text {now }}+c_{n} e_{i}\right)\right) . \\
& l=f\left(\theta_{\text {baseline }} \circ\left(x_{\text {now }}-c_{n} e_{i}\right)\right) . \\
& g=(u-l) /\left(2 c_{n}\right) . \\
& x_{\text {now }}=x_{\text {now }}-a_{n} g .
\end{aligned}
$$

end for

$n=n+1$.

\section{end while}

Output $\theta_{\text {baseline }} \circ x_{\text {now }}$.

(o denotes the Hadamard product).

failure probability, among the worst possible of all eligible $e$ 's, is 0.2732 .

For $\theta_{\text {new }}$, the ranges of $R_{1}(\theta), R_{2}(\theta), R_{3}(\theta)$ and $R(\theta)$ (defined in Section 9.1) are approximately [0, 0.5935], [0, 0.7469], [0, 0.5465] and [0,0.8205]. We could observe from the plots of $R_{\min }$ and $R_{\max }$ that $e_{2}$ has significant different patterns on high values in both plots. According to the trends shown in the plots, we rank the epistemic variables as $e_{2}>e_{3}>e_{1}>e_{4}$.

\section{Design Tuning (Problem E)}

With data sequence $D_{2}=\left\{z^{(i)}(t)\right\}$ for $i=1, \ldots, n_{2}$, we may incorporate the additional information to update our model as before. Similar to Section 7, we use Fourier transform to summarize the highly seasonal responses. In particular, we represent $\left(z_{1}^{(i)}(t)\right)_{t=0, \ldots, T}$ and $\left(z_{2}^{(i)}(t)\right)_{t=0, \ldots, T}$ as $z_{1}^{(i)}(t)=\sum_{k=-\infty}^{\infty} C_{k}^{1} e^{-i k \omega_{0} t}$ and $z_{2}^{(i)}(t)=\sum_{k=-\infty}^{\infty} C_{k}^{2} e^{-i k \omega_{0} t}$ respectively. As shown in Figures 9 and 10 , the responses in frequency domain have common patterns in the positive and negative peaks. Again we use the values of these peaks and their corresponding frequencies to summarize $z_{1}$ and $z_{2}$, which leads to 20 extra parameters adding to the 12 parameters extracted from $D_{1}$.

With the extracted parameters from both $D_{1}$ and $D_{2}$, we now update 


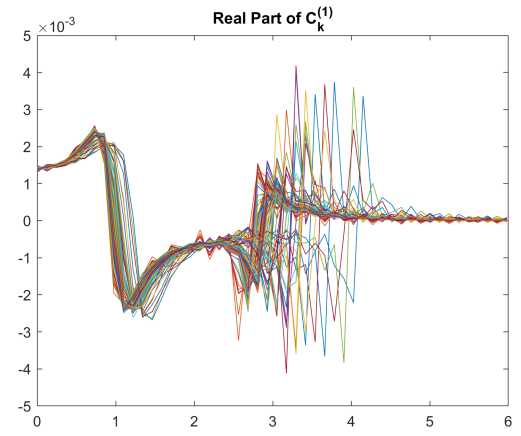

(a) Real part

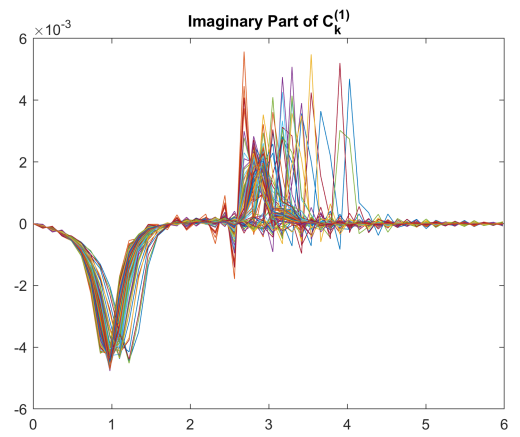

(b) Imaginary part

Figure 9: The real part and the imaginary part pf $C_{k}^{1}$ 's against the corresponding frequencies.

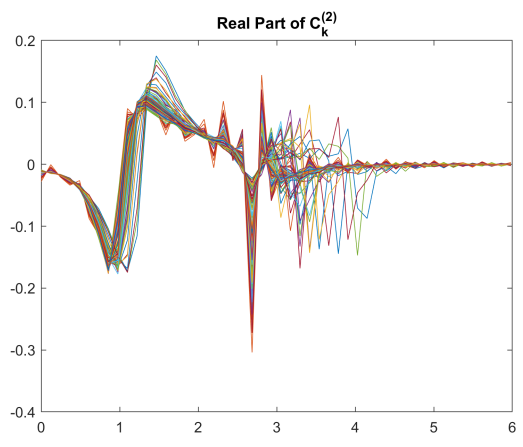

(a) Real part

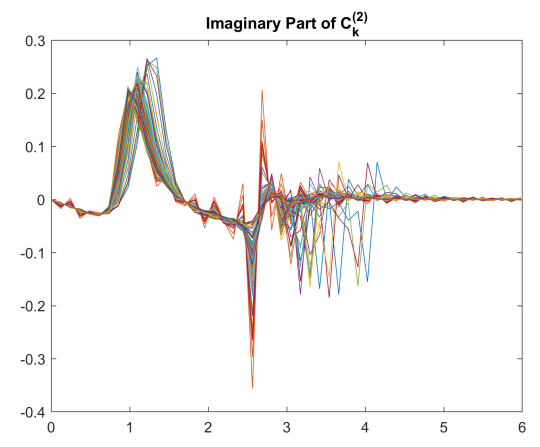

(b) Imaginary part

Figure 10: The real part and the imaginary part pf $C_{k}^{2}$ 's against the corresponding frequencies.

our eligibility set for $E$ by computing $q_{l}^{*}$ 's. We determine eligible $e$ 's with the new threshold $q_{1-0.05 / 32}=1.89$. The values of $q_{l}^{*}$ 's are presented in Figure 11. Compared with Figure 5, we observe that the trend in $e_{2}$ changes slightly. The $q_{l}^{*}$ 's with high value in $e_{2}$ become higher after introducing the information from $D_{2}$, which indicates that $e$ with higher $e_{2}$ is less eligible. Based on the stronger trend in $e_{2}$ and the observation in Section 10, we determine to refine $e_{2}$ on both ends.

In Figure 12, we present $q_{l}^{*}$ 's of samples of $e$ for determining the final eligibility set, $E_{2}$. With these updated information, the final design $\theta_{\text {final }}$ is obtained using Algo. 2, where $\theta_{\text {final }}$ : $(-0.21762,-0.66706,295.61,4410.3$, $4394.1,1.1968,264.49,16.444,127.18)$. The ranges of $R_{1}\left(\theta_{\text {final }}\right), R_{2}\left(\theta_{\text {final }}\right)$, 


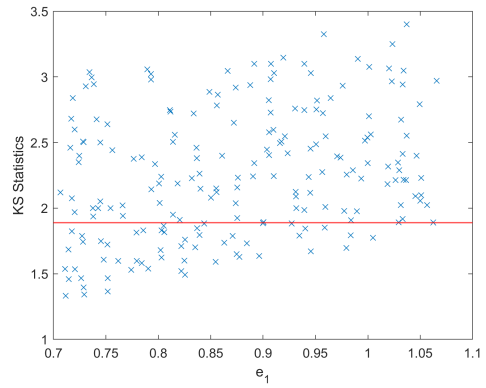

(a) $e_{1}$

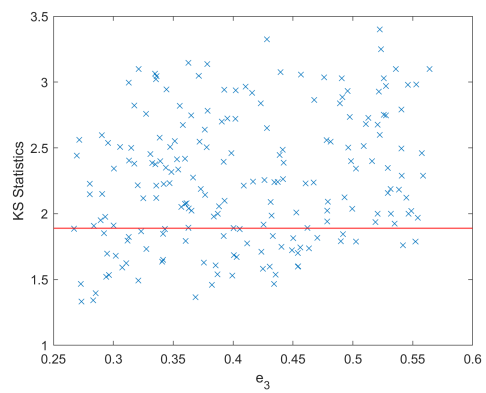

(c) $e_{3}$

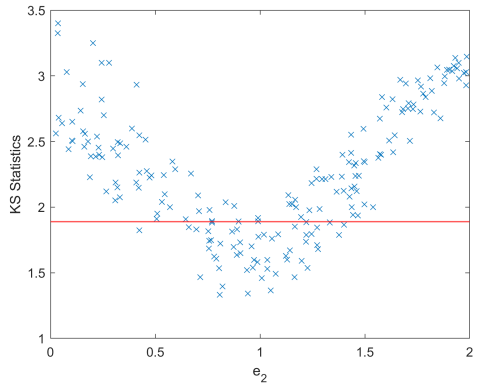

(b) $e_{2}$

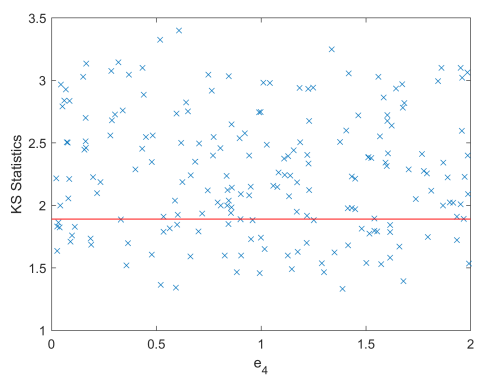

(d) $e_{4}$

Figure 11: $q_{l}^{*}$ against each epistemic variable (after incorporating $D_{2}$ ). $R_{3}\left(\theta_{\text {final }}\right)$ and $R\left(\theta_{\text {final }}\right)$ are $[0,0.1676],[0,0.1620],[0,0.046]$ and $[0,0.2551]$ respectively. Compared to $\theta_{\text {baseline }}$ and $\theta_{\text {new }}$, the worst-case reliability performance is significantly improved.

\section{Risk-Based Design (Problem F)}

Recall that we create an eligibility set for $e$ in the form of $\left\{e^{(l)}: q_{l}^{*} \leq\right.$ $\left.q_{1-\alpha / m}\right\}$, which provides us $(1-\alpha)$ confidence for covering the truth asymptotically. To reduce $r \%$ volume of the eligibility set, we remove $r \%$ number of eligible points in the set with the larger $q_{l}^{*}$ 's. Since larger $q_{l}^{*}$ indicates less similarity with the true response, the reduced eligibility set maintains more important $e^{(l)}$ 's.

In our setting for $e$, taking risks is equivalent to reducing the confidence level for covering the truth. Let us assume the $r \%$ upper quantile of $q_{l}^{*}$ is $q_{r} \%$. Then the reduced eligibility set can be represented as $\left\{e^{(l)}: q_{l}^{*} \leq q_{r} \%\right.$. By finding the $\tilde{\alpha}$ such that $q_{r \%}=q_{1-\tilde{\alpha} / m}$, we can find the confidence level 


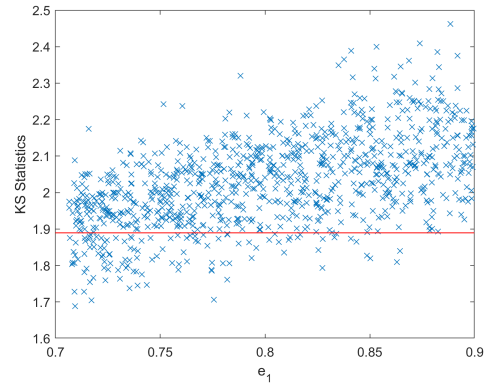

(a) $e_{1}$

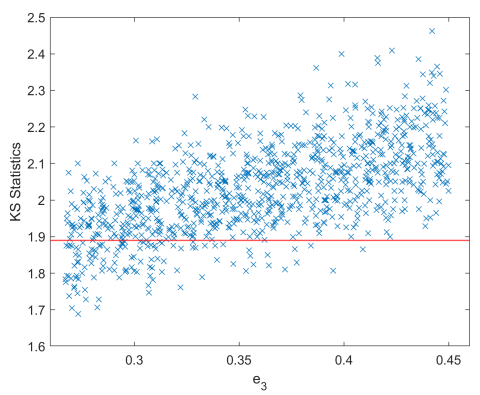

(c) $e_{3}$

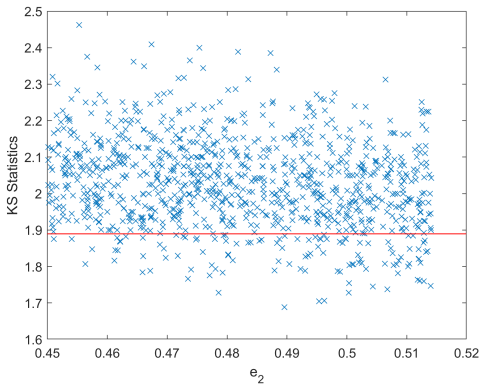

(b) $e_{2}$

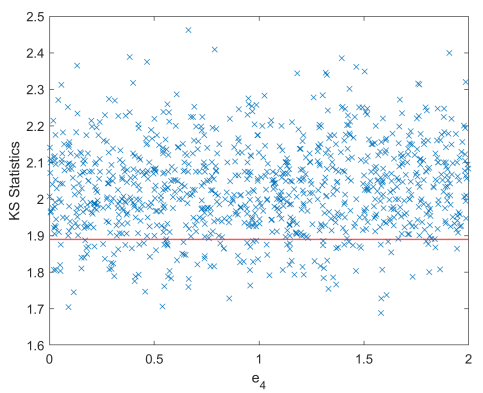

(d) $e_{4}$

Figure 12: $q_{l}^{*}$ against each epistemic variable (final refined).

$1-\tilde{\alpha}$ that corresponds to each choice of $r \%$. In later discussion, the reduced eligibility set corresponding to risk level $r \%$ is denoted as $E_{r \%}$.

In our experiment, we use $E_{2}$ in Section 11 as the baseline. Table 12 shows the risk levels and their corresponding confidence levels. The relation between $r \%$ and $\tilde{\alpha}$ highly depends on the value of $q_{l}^{*}$ 's. In our case, we observe that a large portion of $q_{l}^{*}$ 's are close to $q_{1-\alpha / m}$. As a consequence, the reduction in the volume of the set does not lead to a similar extent of reduction in the confidence level. Since the confidence level is almost not changed, we can anticipate that the design results with different $r \%$ in the range of $(0,10)$ will perform similarly.

With different $r \%$ 's, we construct $E_{r \%}$ 's using the above approach and implement Algo. 2 to obtain risk-based designs $\theta_{r \%}$ 's. Then we evaluate the $\theta_{r \%}$ 's by computing the reliability and severity metrics based on their corresponding eligibility set $E_{r} \%$ and also $E_{2}$. The evaluation results using $E_{2}$ are shown in Figure 13. We observe from Figure 13 that both the reliability or severity metrics are insensitive to the change of $r \%$. In fact, the results are 
Table 1: The risk levels against their corresponding confidence level.

\begin{tabular}{|l|l|l|l|l|l|l|}
\hline$r \%$ & 0 & 2 & 4 & 6 & 8 & 10 \\
\hline$\left|E_{r \%}\right|$ & 114 & 113 & 110 & 108 & 106 & 104 \\
\hline$q_{r \%}$ & 1.89 & 1.886 & 1.884 & 1.884 & 1.881 & 1.879 \\
\hline $1-\tilde{\alpha}$ & $95 \%$ & $94.9 \%$ & $94.8 \%$ & $94.8 \%$ & $94.7 \%$ & $94.6 \%$ \\
\hline
\end{tabular}

also insensitive to whether using $E_{2}$ or $E_{r \%}$ to compute the metrics. Since the difference can be neglected (the largest difference is smaller than 0.01), we omit the results using $E_{r \%}$. From these results, we confirm our conjecture that taking risks would not make much difference in our design approach.

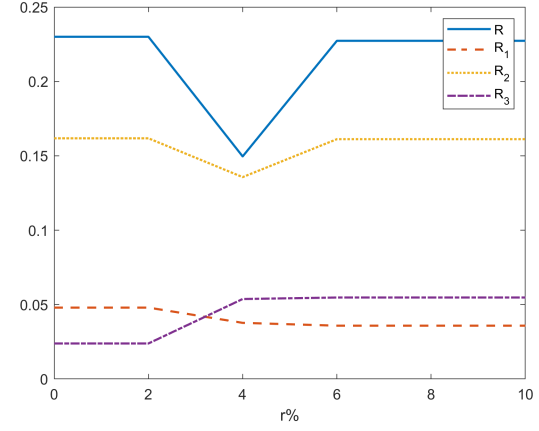

(a) Reliability

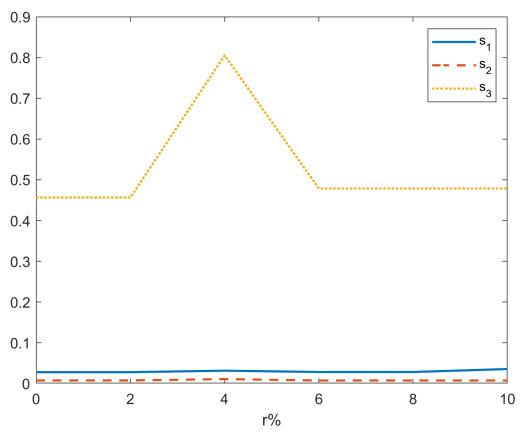

(b) Severity

Figure 13: The reliability and severity metrics for $\theta_{r \%}$ 's evaluated using $E_{2}$.

\section{Discussion}

In this UQ Challenge, we propose a methodology to calibrate model parameters and quantify calibration errors from output data under both aleatory and epistemic uncertainties. The approach utilizes a framework based on an integration of distributionally robust optimization and importance sampling, and operates computationally by solving sampled linear programs. It provides theoretical confidence guarantees on the coverage of the ground truth parameters and distributions. We apply and illustrate our approach to the model calibration and downstream risk analysis tasks in the UQ Challenge. Our approach is drastically different from established Bayesian methodologies, both in the type of guarantee (frequentist versus Bayesian) and computation method (optimization versus posterior sampling). We an- 
ticipate much further work in the future in expanding our methodology to more general problems as well as comparing with the established approaches.

We discuss some immediate future improvement in our implementation in this UQ Challenge. Our procedure relies on several configurations that warrant further explorations. First, the eligibility set geometry is dictated by the choices of the distance metric between distributions and the summary function. Our choice of KS-distance is motivated from nonparametric hypothesis testing that provides asymptotic guarantees. However, since only a finite number of samples is available in practice, its performance can be problem dependent, and other nonparametric test statistics could be considered. Regarding summary functions, we have chosen them based on the visualization of Fourier transform and justify their number via a balance of representativeness and conservativeness in simultaneous estimation. Our refinement results indicate that our eligibility set performs well in locating $e$, which validates our configurations. Nonetheless, a more rigorous approach to choose both the distance metric and the summary functions is desirable.

Our approach requires sampling a number of $a$ and $e$ for eligibility set and aleatory distribution construction. Since a limited size of naive (uniform) sample might miss important information in a large continuous space and cause high variance, we have used several variance reduction techniques including stratified sampling and common random numbers. We note that the samples for $a$ have a larger effects on designs, since they are used to construct the associated best- and worst-case distributions and the quality of samples can be crucial to correctly evaluating the design performances. Moreover, a good sampling scheme can also lead to higher stability of the stochastic gradient descent algorithm.

Lastly, we note that the conservative nature of our robust approach is reflected in the system design. While our robust approach performs well in locating the eligibility set and providing upper bounds on reliability, directly using these bounds as the objectives for optimizing designs appear overconservative. Further work on improving the choice of eligibility sets and sampling on $a$ and $e$ could help improve these design performances.

\section{Appendix A. Proof of Theorem 2}

This proof is adapted from [29]. We denote $L=d P_{\text {true }} / d P_{0}$. Let $W_{j}=$ $\frac{L\left(a^{(j)}\right)}{\sum_{j=1}^{k} L\left(a^{(j)}\right)}$. For simplicity, we use $\mathbf{y}(a)$ to denote $\left(y\left(a, e_{t r u e}, t\right)\right)_{t=1, \ldots, T}$ and 
use $\mathbf{y}_{j}$ to denote $\left(y\left(a^{(j)}, e_{\text {true }}, t\right)\right)_{t=1, \ldots, T}$. Then we have that

$$
\begin{aligned}
& \sup _{x \in \mathbb{R}}\left|\sum_{j=1}^{k} W_{j} I\left(S_{v}\left(\mathbf{y}_{j}\right) \leq x\right)-\hat{F}_{v}(x)\right| \\
\leq & \sup _{x \in \mathbb{R}}\left|\sum_{j=1}^{k} W_{j} I\left(S_{v}\left(\mathbf{y}_{j}\right) \leq x\right)-\frac{1}{k} \sum_{j=1}^{k} L\left(a^{(j)}\right) I\left(S_{v}\left(\mathbf{y}_{j}\right) \leq x\right)\right|+ \\
& \sup _{x \in \mathbb{R}}\left|\frac{1}{k} \sum_{j=1}^{k} L\left(a^{(j)}\right) I\left(S_{v}\left(\mathbf{y}_{j}\right) \leq x\right)-E_{P_{0}}\left(L(a) I\left(S_{v}(\mathbf{y}(a)) \leq x\right)\right)\right|+ \\
& \sup _{x \in \mathbb{R}}\left|E_{P_{\text {true }}}\left(I\left(S_{v}(\mathbf{y}(a)) \leq x\right)\right)-\hat{F}_{v}(x)\right| .
\end{aligned}
$$

For the first term, we have that

$$
\begin{aligned}
& \sum_{j=1}^{k} W_{j} I\left(S_{v}\left(\mathbf{y}_{j}\right) \leq x\right)-\frac{1}{k} \sum_{j=1}^{k} L\left(a^{(j)}\right) I\left(S_{v}\left(\mathbf{y}_{j}\right) \leq x\right) \\
= & \frac{1}{k} \sum_{j=1}^{k} L\left(a^{(j)}\right) I\left(S_{v}\left(\mathbf{y}_{j}\right) \leq x\right)\left(\frac{k}{\sum_{j=1}^{k} L\left(a^{(j)}\right)}-1\right) .
\end{aligned}
$$

Since $\left\|d P_{\text {true }} / d P_{0}\right\|_{\infty} \leq C$, we get that $\frac{1}{k} \sum_{j=1}^{k} L\left(a^{(j)}\right) I\left(S_{v}\left(\mathbf{y}_{j}\right) \leq x\right) \leq C$. Moreover, we know that $E_{P_{0}}(L)=1$ and $\operatorname{var}_{P_{0}}(L)<\infty$, and thus

$$
\sqrt{k}\left(\frac{k}{\sum_{j=1}^{k} L\left(a^{(j)}\right)}-1\right) \Rightarrow N\left(0, \operatorname{var}_{P_{0}}(L)\right) .
$$

Hence, we get that

$$
\sup _{x \in \mathbb{R}}\left|\sum_{j=1}^{k} W_{j} I\left(S_{v}\left(\mathbf{y}_{j}\right) \leq x\right)-\frac{1}{k} \sum_{j=1}^{k} L\left(a^{(j)}\right) I\left(S_{v}\left(\mathbf{y}_{j}\right) \leq x\right)\right|=O_{p}(1 / \sqrt{k}) .
$$

For the second term, following the proof in [29], we know that

$$
\left\{\sqrt{k}\left(\frac{1}{k} \sum_{j=1}^{k} L\left(a^{(j)}\right) I\left(S_{v}\left(\mathbf{y}_{j}\right) \leq x\right)-E_{P_{0}}\left(L(a) I\left(S_{v}(\mathbf{y}(a)) \leq x\right)\right)\right)\right\} \Rightarrow\{G(x)\}
$$


in $\ell^{\infty}\left(\left\{a \mapsto L(a) I\left(S_{v}(\mathbf{y}(a)) \leq x\right): x \in \mathbb{R}\right\}\right)$ and $G$ is a Gaussian process. Therefore, we get that

$$
\sup _{x \in \mathbb{R}}\left|\frac{1}{k} \sum_{j=1}^{k} L\left(a^{(j)}\right) I\left(S_{v}\left(\mathbf{y}_{j}\right) \leq x\right)-E_{P_{0}}\left(L(a) I\left(S_{v}(\mathbf{y}(a)) \leq x\right)\right)\right|=O_{p}(1 / \sqrt{k}) .
$$

Finally, it is known that

$$
\sqrt{n_{1}} \sup _{x \in \mathbb{R}}\left|E_{P_{\text {true }}}\left(I\left(S_{v}(\mathbf{y}(a)) \leq x\right)\right)-\hat{F}_{v}(x)\right| \Rightarrow \sup _{x \in[0,1]}\left|B B\left(F_{\text {true }, r}(x)\right)\right| .
$$

Combining the above results, we get that for each $v=1, \ldots, m$,

$$
\limsup _{n_{1} \rightarrow \infty, k / n_{1} \rightarrow \infty} \mathbb{P}\left(\sup _{x \in \mathbb{R}}\left|\sum_{j=1}^{k} W_{j} I\left(S_{v}\left(\mathbf{y}_{j}\right) \leq x\right)-\hat{F}_{v}(x)\right|>\frac{q_{1-\alpha / m}}{\sqrt{n_{1}}}\right) \leq \frac{\alpha}{m}
$$

and hence

$$
\liminf _{n_{1} \rightarrow \infty, k / n_{1} \rightarrow \infty} \mathbb{P}\left(e_{\text {true }} \in E\right) \geq 1-\alpha .
$$

\section{Acknowledgements}

We gratefully acknowledge support from the National Science Foundation under grants CAREER CMMI-1834710 and IIS-1849280. A preliminary conference version of this paper has appeared in the Proceedings of the 30th European Safety and Reliability Conference and the 15th Probabilistic Safety Assessment and Management Conference (ESREL-PSAM) 2020.

\section{References}

[1] Asmussen, S. and P. W. Glynn (2007). Stochastic simulation: algorithms and analysis, Volume 57. Springer Science \& Business Media.

[2] Balci, O. and R. G. Sargent (1982). Some examples of simulation model validation using hypothesis testing. In H. J. Highland, Y. W. Chao, and O. S. Madrigal (Eds.), Proceedings of the 14 th Conference on Winter Simulation - Volume 2, Piscataway, New Jersey, pp. 621-629. Institute of Electrical and Electronics Engineers, Inc. 
[3] Bayarri, M. J., J. O. Berger, R. Paulo, J. Sacks, J. A. Cafeo, J. Cavendish, C.-H. Lin, and J. Tu (2007). A framework for validation of computer models. Technometrics 49(2), 138-154.

[4] Bayraksan, G. and D. K. Love (2015). Data-driven stochastic programming using phi-divergences. In Tutorials in Operations Research, pp. 1-19. INFORMS.

[5] Ben-Tal, A., D. Den Hertog, A. De Waegenaere, B. Melenberg, and G. Rennen (2013). Robust solutions of optimization problems affected by uncertain probabilities. Management Science 59(2), 341-357.

[6] Ben-Tal, A. and A. Nemirovski (2002). Robust optimizationmethodology and applications. Mathematical Programming 92(3), 453480 .

[7] Bertsimas, D., D. B. Brown, and C. Caramanis (2011). Theory and applications of robust optimization. SIAM Review 53(3), 464-501.

[8] Bertsimas, D., V. Gupta, and N. Kallus (2018a). Data-driven robust optimization. Mathematical Programming 167(2), 235-292.

[9] Bertsimas, D., V. Gupta, and N. Kallus (2018b, September). Robust sample average approximation. 171(1-2), 217-282.

[10] Bertsimas, D., V. Gupta, and N. Kallus (2018c). Robust sample average approximation. Mathematical Programming 171(1-2), 217-282.

[11] Blanchet, J., Y. Kang, and K. Murthy (2019). Robust wasserstein profile inference and applications to machine learning. Journal of Applied Probability 56(3), 830-857.

[12] Blanchet, J. and H. Lam (2012). State-dependent importance sampling for rare-event simulation: An overview and recent advances. Surveys in Operations Research and Management Science 17(1), 38-59.

[13] Blanchet, J. and K. R. Murthy (2016). Quantifying distributional model risk via optimal transport. arXiv preprint arXiv:1604.01446.

[14] Bucklew, J. (2013). Introduction to Rare Event Simulation. New York: Springer Science \& Business Media. 
[15] Craig, P. S., M. Goldstein, J. C. Rougier, and A. H. Seheult (2001). Bayesian forecasting for complex systems using computer simulators. Journal of the American Statistical Association 96(454), 717-729.

[16] Crespo, L. and S. Kenny (2020). The NASA Langley Challenge on Optimization under Uncertainty. ESREL.

[17] Currin, C., T. Mitchell, M. Morris, and D. Ylvisaker (1991). Bayesian prediction of deterministic functions, with applications to the design and analysis of computer experiments. Journal of the American Statistical Association 86(416), 953-963.

[18] Delage, E. and Y. Ye (2010). Distributionally robust optimization under moment uncertainty with application to data-driven problems. Operations research 58(3), 595-612.

[19] Duchi, J. C., P. W. Glynn, and H. Namkoong (0). Statistics of robust optimization: A generalized empirical likelihood approach. Mathematics of Operations Research 0(0), null.

[20] Esfahani, P. M. and D. Kuhn (2018). Data-driven distributionally robust optimization using the wasserstein metric: Performance guarantees and tractable reformulations. Mathematical Programming 171(1-2), 115-166.

[21] Fu, M. C. (2015). Handbook of simulation optimization, Volume 216. Springer.

[22] Gao, R. and A. J. Kleywegt (2016). Distributionally robust stochastic optimization with Wasserstein distance. arXiv preprint arXiv:1604.02199.

[23] Ghadimi, S. and G. Lan (2013). Stochastic first-and zeroth-order methods for nonconvex stochastic programming. SIAM Journal on Optimization 23(4), 2341-2368.

[24] Ghaoui, L. E., M. Oks, and F. Oustry (2003). Worst-case value-atrisk and robust portfolio optimization: A conic programming approach. Operations research 51(4), 543-556.

[25] Ghosh, S. and H. Lam (2019). Robust analysis in stochastic simulation: Computation and performance guarantees. Operations Research 67(1), 232-249. 
[26] Glasserman, P. (2013). Monte Carlo methods in financial engineering, Volume 53. Springer Science \& Business Media.

[27] Glasserman, P. and X. Xu (2014). Robust risk measurement and model risk. Quantitative Finance 14(1), 29-58.

[28] Glynn, P. W. and D. L. Iglehart (1989). Importance sampling for stochastic simulations. Management science 35(11), 1367-1392.

[29] Goeva, A., H. Lam, H. Qian, and B. Zhang (2019). Optimization-based calibration of simulation input models. Operations Research 67(5), 13621382 .

[30] Goh, J. and M. Sim (2010). Distributionally robust optimization and its tractable approximations. Operations Research 58(4-Part-1), 902-917.

[31] Gupta, V. (2019). Near-optimal bayesian ambiguity sets for distributionally robust optimization. Management Science 65(9), 4242-4260.

[32] Higdon, D., J. Gattiker, B. Williams, and M. Rightley (2008). Computer model calibration using high-dimensional output. Journal of the American Statistical Association 103(482), 570-583.

[33] Hong, L. J., Z. Huang, and H. Lam (2020). Learning-based robust optimization: Procedures and statistical guarantees. Management Science.

[34] Hu, Z., J. Cao, and L. J. Hong (2012). Robust simulation of global warming policies using the dice model. Management Science 58(12), 21902206 .

[35] Jian, N. and S. G. Henderson (2015). An introduction to simulation optimization. In 2015 winter simulation conference (wsc), pp. 1780-1794. IEEE.

[36] Juneja, S. and P. Shahabuddin (2006). Rare-event simulation techniques: an introduction and recent advances. Handbooks in operations research and management science 13, 291-350.

[37] Kennedy, M. C. and A. O'Hagan (2001). Bayesian calibration of computer models. Journal of the Royal Statistical Society: Series B (Statistical Methodology) 63(3), 425-464. 
[38] Kleijnen, J. P. (1995). Verification and validation of simulation models. European Journal of Operational Research 82(1), 145-162.

[39] Kraan, B. and T. Bedford (2005). Probabilistic inversion of expert judgments in the quantification of model uncertainty. Management science 51(6), 995-1006.

[40] Kushner, H. and G. G. Yin (2003). Stochastic approximation and recursive algorithms and applications, Volume 35. Springer Science \& Business Media.

[41] Lam, H. (2016). Robust sensitivity analysis for stochastic systems. Mathematics of Operations Research 41(4), 1248-1275.

[42] Lam, H. (2018). Sensitivity to serial dependency of input processes: A robust approach. Management Science 64(3), 1311-1327.

[43] Lam, H. (2019). Recovering best statistical guarantees via the empirical divergence-based distributionally robust optimization. Operations Research $67(4), 1090-1105$.

[44] Lam, H. and H. Qian (2019). Combating conservativeness in data-driven optimization under uncertainty: A solution path approach. arXiv preprint arXiv:1909.0647\%.

[45] Lam, H. and E. Zhou (2017). The empirical likelihood approach to quantifying uncertainty in sample average approximation. Operations Research Letters 45(4), $301-307$.

[46] Li, B., R. Jiang, and J. L. Mathieu (2017). Ambiguous risk constraints with moment and unimodality information. Mathematical Programming, $1-42$.

[47] Liu, J. S. (2008). Monte Carlo strategies in scientific computing. Springer Science \& Business Media.

[48] Marjoram, P., J. Molitor, V. Plagnol, and S. Tavaré (2003). Markov chain monte carlo without likelihoods. Proceedings of the National Academy of Sciences 100(26), 15324-15328.

[49] Nelson, B. L. (2016). 'Some Tactical Problems in Digital Simulation' for the Next 10 Years. Journal of Simulation 10(1), 2-11. 
[50] Pan, S. J. and Q. Yang (2009). A survey on transfer learning. IEEE Transactions on knowledge and data engineering 22(10), 1345-1359.

[51] Petersen, I. R., M. R. James, and P. Dupuis (2000). Minimax optimal control of stochastic uncertain systems with relative entropy constraints. IEEE Transactions on Automatic Control 45(3), 398-412.

[52] Popescu, I. (2005). A semidefinite programming approach to optimalmoment bounds for convex classes of distributions. Mathematics of Operations Research 30(3), 632-657.

[53] Precup, D. (2000). Eligibility traces for off-policy policy evaluation. Computer Science Department Faculty Publication Series, 80.

[54] Rubinstein, R. Y. and D. P. Kroese (2016). Simulation and the Monte Carlo method, Volume 10. John Wiley \& Sons.

[55] Sargent, R. G. (2010). Verification and validation of simulation models. In S. Jain, R. R. Creasey, J. Himmelspach, K. P. White, and M. Fu (Eds.), Proceedings of the 2010 winter simulation conference, Piscataway, New Jersey, pp. 166-183. Institute of Electrical and Electronics Engineers, Inc.

[56] Schlegel, M., W. Chung, D. Graves, J. Qian, and M. White (2019). Importance resampling for off-policy prediction. In Advances in Neural Information Processing Systems, pp. 1799-1809.

[57] Schruben, L. W. (1980). Establishing the Credibility of Simulations. Simulation 34(3), 101-105.

[58] Siegmund, D. (1976). Importance sampling in the monte carlo study of sequential tests. The Annals of Statistics, 673-684.

[59] Sugiyama, M., S. Nakajima, H. Kashima, P. Von Buenau, and M. Kawanabe (2007). Direct importance estimation with model selection and its application to covariate shift adaptation. In NIPS, Volume 7, pp. 1433-1440. Citeseer.

[60] Tarantola, A. (2005). Inverse Problem Theory and Methods for Model Parameter Estimation. Philadelphia, Pennsylvania: Society for Industrial and Applied Mathematics. 
[61] Van Parys, B. P., P. J. Goulart, and D. Kuhn (2016). Generalized gauss inequalities via semidefinite programming. Mathematical Programming 156(1-2), 271-302.

[62] Wiesemann, W., D. Kuhn, and M. Sim (2014). Distributionally robust convex optimization. Operations Research 62(6), 1358-1376. 